\title{
Curves of Restricted Type in Euclidean Spaces
}

\author{
Bengü (KILIÇ) Bayram And Nergiz Önen
}

\begin{abstract}
Submanifolds of restricted type were introduced in [7]. In the present study we consider restricted type of curves in $\mathbb{E}^{m}$. We give some special examples. We also show that spherical curve in $S^{2}(r) \subset \mathbb{E}^{3}$ is of restricted type if and only if either $f(s)$ is constant or a linear function of $s$ of the form $f(s)= \pm s+b$ and every closed $W$-curve of rank $k$ and of length $2 \pi r$ in $\mathbb{E}^{2 k}$ is of restricted type.
\end{abstract}

\section{INTRODUCTION}

Let $M^{n}$ be an $n$-dimensional submanifold of a Euclidean space $\mathbb{E}^{m}$. Let $x, H$ and $\Delta$ respectively be the position vector field, the mean curvature vector field and the Laplace operator of the induced metric on $M^{n}$. Then, as is well known (see e.g. [2])

$$
\Delta x=-n H,
$$

which shows, in particular, that $M^{n}$ is a minimal submanifold in $\mathbb{E}^{m}$ if and only if its coordinate functions are harmonic (i.e. they are eigenfunctions of $\Delta$ with eigenvalue 0 ).

As a generalization of T. Takahashi's condition and following an idea of O. Garay [13], some of the authors together with J. Pas [10] initiated the study of submanifolds $M^{n}$ in $\mathbb{E}^{m}$ such that

$$
\Delta x=A x+B
$$

for some fixed vector $B \in \mathbb{E}^{m}$ and a given matrix $A \in \mathbb{R}^{m \times m}$. This study was continued by the first author together with $M$. Petrovic [5] and independently by T. Hasanis and T. Vlachos [14].

During the study of submanifolds of $\mathbb{R}^{m}$ satisfying (2), it was observed that all these matrices $A_{p}$ are equal for all $p \in M$, or equivalently there exists a fixed matrix $A \in \mathbb{E}^{m \times m}$ (determining, of course, a linear endomorphism of $\mathbb{E}^{m}$ ) such that for all $p \in M$ and for all $X \in T_{p} M$,

$$
A_{H} X=(A X)^{T} \text {. }
$$

2010 Mathematics Subject Classification. Primary: 53A04.

Key words and phrases. Curves of restricted type. 
As the relation (3) expresses a strong relationship between differential geometry and linear algebra, we do think it would be worthwhile to study submanifolds satisfying this condition; such submanifolds are said to be of restricted type.

Submanifolds of restricted type were introduced in [7] by the author B.Y. Chen, F. Dillen, L. Verstraelen and L. Vrancken. The class of submanifolds of restricted type is large which includes 1-type submanifolds, pseudoumbilical submanifolds with constant mean curvature, submanifolds satisfying either Gray's condition or Dillen Pas Verstraelen's condition, all $k$-type curves lying fully in $\mathbb{E}^{2 k}$, all null $k$-type curves lying fully in $\mathbb{E}^{2 k-1}$, the products of submanifolds of restricted type, the diagonal immersions of restricted type submanifolds and equivariant isometric immersions of compact homogeneous spaces. In [7], it is shown that a hypersurface of restricted type is either minimal, or a part of the product of a sphere and a linear subspace, or a cylinder on a plane curve of restricted type, and all planar curves of restricted type are classified.

\section{BAsic Concepts}

In the present section we recall definitions and results of [1]. Let $x$ : $M \rightarrow \mathbb{E}^{m}$ be an immersion from an $n$-dimensional connected Riemannian manifold $M$ into an $m$-dimensional Euclidean space $\mathbb{E}^{m}$. We denote by $g$ the metric tensor of $\mathbb{E}^{m}$ as well as the induced metric on $M$. Let $\tilde{\nabla}$ be the Levi-Civita connection of $\mathbb{E}^{m}$ and $\nabla$ the induced connection on $M$. Then the Gaussian and Weingarten formulas are given, respectively, by

$$
\begin{gathered}
\tilde{\nabla}_{X} Y=\nabla_{X} Y+h(X, Y), \\
\tilde{\nabla}_{X} \xi=-A_{\xi} X+D_{X} \xi,
\end{gathered}
$$

where $X, Y$ are vector fields tangent to $M$ and $\xi$ normal to $M$. Moreover, $h$ is the second fundamental form, $D$ is the linear connection induced in the normal bundle $T^{\perp} M$, called normal connection and $A_{\xi}$ the shape operator in the direction of $\xi$ that is related with $h$ by

$$
\langle h(X, Y), \xi\rangle=\left\langle A_{\xi} X, Y\right\rangle .
$$

For an $n$-dimensional submanifold $M$ in $\mathbb{E}^{m}$. The mean curvature vector $\vec{H}$ is given by

$$
\vec{H}=\frac{1}{n} \text { traceh. }
$$

A submanifold $M$ is said to be minimal (respectively, totally geodesic) if $\vec{H} \equiv 0$ (respectively, $h \equiv 0$ ).

Consider an $n$-dimensional Riemannian manifold $M$ and denote by $\left(g_{i j}\right)$ the local components of its metric. Put $G=\operatorname{det}\left(g_{i j}\right)$ and $\left(g^{i j}\right)=\left(g_{i j}\right)^{-1}$. 
Then the Laplacian $\Delta$ of the metric $g$ can be locally defined by

$$
\Delta u=-\frac{1}{\sqrt{G}} \sum_{i, j=1}^{n} \frac{\partial}{\partial x_{i}}\left(\sqrt{G} g^{i j} \frac{\partial u}{\partial x_{j}}\right),
$$

for any function $u$ on $M$, where $x_{1}, x_{2}, \ldots, x_{n}$ are local coordinates [11].

$M$ is said to be of finite type if each component of the position vector $x$ has a finite spectral decomposition [2]

$$
x=x_{0}+x_{1}+x_{2}+\cdots+x_{k},
$$

where $x_{0}$ is a constant vector in $\mathbb{E}^{m}$ and $x_{1}, x_{2}, \ldots, x_{k}$ are non-constant maps which satisfy $\Delta x_{i}=\lambda_{i} x_{i}, 1 \leq i \leq k, \lambda_{1}<\lambda_{2}<\cdots<\lambda_{k}$.

If all eigenvalues $\lambda_{1}, \lambda_{2}, \ldots, \lambda_{k}$ are mutually distinct, then the immersion $x$ (or the submanifold $M$ ) is said to be of $k$-type [2].

\section{W-CURVES IN $\mathbb{E}^{m}$}

Let $\gamma=\gamma(t): I \rightarrow \mathbb{E}^{m}$ be a regular curve in $\mathbb{E}^{m}$ (i.e. $\left\|\gamma^{\prime}\right\|$ is nowhere zero), where $I$ is interval in $\mathbb{R}$. $\gamma$ is called a Frenet curve of rank $r\left(r \in \mathbb{N}_{0}, r \leq m\right)$ if $\gamma^{\prime}(t), \gamma^{\prime \prime}(t), \ldots, \gamma^{(r)}(t)$ are linearly independent and $\gamma^{\prime}(t), \gamma^{\prime \prime}(t), \ldots, \gamma^{(r+1)}(t)$ are no longer linearly independent for all $t$ in $I$. In this case, $\operatorname{Im}(\gamma)$ lies in an $r$-dimensional Euclidean subspace of $\mathbb{E}^{m}$. To each Frenet curve of rank $r$ there can be associated orthonormal $r$-frame $\left\{V_{1}, V_{2}, \ldots, V_{r}\right\}$ along $\gamma$, the Frenet $r$-frame and $r-1$ functions $\kappa_{1}, \kappa_{2}, \ldots, \kappa_{r-1}: I \rightarrow \mathbb{R}$, the Frenet curvatures, such that

$$
\left[\begin{array}{c}
V_{1}^{\prime} \\
V_{2}^{\prime} \\
V_{3}^{\prime} \\
\vdots \\
V_{r-1}^{\prime} \\
V_{r}^{\prime}
\end{array}\right]=v\left[\begin{array}{cccccc}
0 & \kappa_{1} & 0 & \cdots & 0 & 0 \\
-\kappa_{1} & 0 & \kappa_{2} & \cdots & 0 & 0 \\
0 & -\kappa_{2} & 0 & \cdots & 0 & 0 \\
\vdots & \vdots & \vdots & \ddots & \vdots & \vdots \\
0 & 0 & \cdots & \cdots & 0 & \kappa_{r-1} \\
0 & 0 & \cdots & \cdots & -\kappa_{r-1} & 0
\end{array}\right] \cdot\left[\begin{array}{c}
V_{1} \\
V_{2} \\
V_{3} \\
\vdots \\
V_{r-1} \\
V_{r}
\end{array}\right],
$$

where $v$ is the speed of the curve.

In fact, to obtain $V_{1}, V_{2}, \ldots, V_{r}$ it is sufficient to apply the Gram-Schmidt orthonormalization process to $\gamma^{\prime}(t), \gamma^{\prime}(t), \ldots, \gamma^{(r)}(t)$. Moreover, the functions $\kappa_{1}, \kappa_{2}, \ldots, \kappa_{r-1}$ are easily obtained as by-product during this calculation. More precisely, $V_{1}, V_{2}, \ldots, V_{r}$ and $\kappa_{1}, \kappa_{2}, \ldots, \kappa_{r-1}$ are determined by 
the following formulas:

$$
\begin{aligned}
E_{1}(t) & :=\gamma^{\prime}(t) ; \quad V_{1}:=\frac{E_{1}(t)}{\left\|E_{1}(t)\right\|} \\
E_{k}(t) & :=\gamma^{(k)}(t)-\sum_{i=1}^{k-1}\left\langle\gamma^{(k)}(t), E_{i}(t)\right\rangle \frac{E_{i}(t)}{\left\|E_{i}(t)\right\|} \\
\kappa_{k-1}(t) & :=\frac{E_{k}(t)}{\left\|E_{k-1}(t) E_{1}(t)\right\|} \\
V_{k} & :=\frac{E_{k}(t)}{\left\|E_{k}(t)\right\|}
\end{aligned}
$$

where $k \in\{2,3, \ldots, r\}$. It is natural and convenient to define Frenet curvatures $\kappa_{r}=\kappa_{r+1}=\cdots=\kappa_{m-1}=0$. It is clear that $V_{1}, V_{2}, \ldots, V_{r}$ and $\kappa_{1}, \kappa_{2}, \ldots, \kappa_{r-1}$ can be defined for any regular curve (not necessary a Frenet curve) in the neighborhood of a point $t_{0}$ for which $\gamma^{\prime}\left(t_{0}\right), \gamma^{\prime \prime}\left(t_{0}\right), \ldots, \gamma^{(r)}\left(t_{0}\right)$ are linearly independent.

Definition 1. Frenet curve of rank $r$ for which $\kappa_{1}, \kappa_{2}, \ldots, \kappa_{r-1}$ are constant is called (generalized) screw line or helix [6]. Since these curves are trajectories of the 1-parameter group of the Euclidean transformations, so, F. Klein and S. Lie [9] called them $W$-curves.

A unit speed $W$-curve of rank $2 k$ has the parametrization form

$$
\gamma(s)=a_{0}+\sum_{i=1}^{k}\left(a_{i} \cos \mu_{i} s+b_{i} \sin \mu_{i} s\right),
$$

and a unit speed $W$-curve of rank $(2 k+1)$ has the parametrization form

$$
\gamma(s)=a_{0}+b_{0} s+\sum_{i=1}^{k}\left(a_{i} \cos \mu_{i} s+b_{i} \sin \mu_{i} s\right),
$$

where $a_{0}, b_{0}, a_{1}, \ldots, a_{k}, b_{1}, \ldots, b_{k}$ are constant vectors in $\mathbb{E}^{m}$ and $\mu_{1}<\mu_{2}<$ $\cdots<\mu_{k}$ are positive real numbers.

So, a $W$-curve of rank 1 is a straight line, a $W$-curve of rank 2 is a circle and a $W$-curve of rank 3 is a right circular helix [6].

A $W$-curve is closed if and only if its rank is even and all $\mu_{i}$ are rational multiples of a real number. Therefore, up to rigid motions of a Euclidean space, a closed $W$-curve of rank $2 k$ and of length $2 \pi r$ in $\mathbb{E}^{2 k}$ has an arc length parameterization of the form:

$$
\gamma(s)=\frac{r}{\sqrt{k}}\left(\frac{1}{t_{1}} \cos \left(\frac{t_{1} s}{r}\right), \frac{1}{t_{1}} \sin \left(\frac{t_{1} s}{r}\right), \ldots, \frac{1}{t_{k}} \cos \left(\frac{t_{k} s}{r}\right), \frac{1}{t_{k}} \sin \left(\frac{t_{k} s}{r}\right)\right)
$$

where $t_{1}<\cdots<t_{k}$ are positive integers [8]. 


\section{Curves of Restricted type}

Definition 2. A submanifold $M^{n}$ in $\mathbb{E}^{m}$ is said to be restricted type if the shape operator $A_{H}$ is the restriction of a fixed endomorphism $A$ of $\mathbb{E}^{m}$ on the tangent space of $M^{n}$ at every point of $M^{n}$, i.e.

$$
A_{H} X=(A X)^{T}
$$

for any vector $X$, tangent to $M^{n}$, where $(A X)^{T}$ denotes the tangential component of $A X[7]$.

Remark 1. Equation (14) is equivalent to $\left\langle A_{H} X, Y\right\rangle=\langle A X, Y\rangle$ for all tangent vectors $X, Y[7]$.

Proposition 1. Every submanifold $M^{n}$ in $\mathbb{E}^{m}$ whose position vector field satisfies $\Delta x=\tilde{A} x+B$, where $\Delta$ is the Laplacian of $M^{n}, \tilde{A} \in \mathbb{R}^{m \times m}$ and $B \in \mathbb{E}^{m}$, is of restricted type. The endomorphism $A$ is given by $\frac{1}{n} \tilde{A}$ in this case [7].

Let $\gamma$ be a regular curve in $\mathbb{E}^{m}$. The Laplacian of $\gamma$ can be expressed as

$$
\Delta \gamma(t)=-\frac{d^{2} \gamma(t)}{d t^{2}}=-\gamma^{\prime \prime}(t)
$$

By the using of (1) and (15),

$$
H=-\Delta \gamma(t)=\gamma^{\prime \prime}(t)
$$

where $H$ is the mean curvature of $\gamma$.

Proposition 2. Let $\gamma$ be a curve in $\mathbb{E}^{m}$. If $\gamma$ has the equation

$$
-\gamma^{\prime \prime}(t)=\Delta \gamma(t)=A \gamma(t)+B
$$

such that $B$ is a fixed vector in $\mathbb{E}^{m}$ and $A$ a symmetric matrix in $\mathbb{R}^{m \times m}$, then $\gamma$ is of restricted type.

Proof. From Preposition 1 we have the equation

$$
\Delta \gamma(t)=A \gamma(t)+B \text {. }
$$

Thus using (16) and (18), we get (17).

Corollary 1. Let $\gamma$ be a curve in $\mathbb{E}^{m} \cdot \gamma$ is of restricted type if and only if

$$
-\gamma^{\prime \prime \prime}(t)=A \gamma^{\prime}(t)
$$

where $A$ is a symmetric matrix in $\mathbb{R}^{m \times m}$.

Example 1. $S^{1}(a) \subset \mathbb{E}^{2}$ is of restricted type.

$S^{1}(a)$ is given by the parametrization $\gamma(t)=(a \cos t, a \sin t)$. From higher order derivatives of $\gamma$ we get

$$
\gamma^{\prime \prime \prime}(t)=-I_{2} \gamma^{\prime}(t) .
$$

Thus $S^{1}(a) \subset \mathbb{E}^{2}$ is of restricted type. 
Example 2. A helix which is given by the parametrization

$$
\gamma(t)=(r \cos (c t+d), r \sin (c t+d), a t+b)
$$

is of restricted type.

From higher order derivatives of $\gamma$ we get $\gamma^{\prime \prime \prime}(t)=-A \gamma^{\prime}(t)$ where

$$
A=\left[\begin{array}{ccc}
c^{2} & 0 & 0 \\
0 & c^{2} & 0 \\
0 & 0 & 0
\end{array}\right]
$$

Thus helix is of restricted type.

Example 3. Every $k$-type curve which lies fully in $\mathbb{E}^{2 k}$ is of restricted type [7].

Example 4. Every 2-type curve in $\mathbb{E}^{m}$ is of restricted type [7].

Example 5. Although every 2-type curve in $\mathbb{E}^{m}$ and every $k$-type curve which lies fully in $\mathbb{E}^{2 k}$ are curves of restricted type, not every curve of finite type (in the sense of $[2,4]$ ) is of restricted type. For instance the following 6 -type curve in $\mathbb{E}^{3}$ is not of restricted type [7]

$$
\begin{aligned}
\gamma(s)= & \left(-\frac{2}{3} \cos \frac{12}{17} s+\frac{3}{4} \cos \frac{16}{17} s+\frac{3}{10} \cos \frac{20}{17} s+\frac{1}{8} \cos \frac{24}{17} s+\frac{1}{14} \cos \frac{28}{17} s,\right. \\
& \left.-\frac{2}{3} \sin \frac{12}{17} s+\frac{3}{4} \sin \frac{16}{17} s+\frac{3}{10} \sin \frac{20}{17} s+\frac{1}{8} \sin \frac{24}{17} s+\frac{1}{14} \sin \frac{28}{17} s, \sin \frac{8}{17} s\right) .
\end{aligned}
$$

Proposition 3. Let $\gamma$ be a spherical space curve given with

$$
\gamma(s)=(r \cos s \sin (f(s)), r \sin s \sin (f(s)), r \cos (f(s)),
$$

where $f(s)$ is polynomial function. Then $\gamma$ is of restricted type if and only if $f(s)$ is either constant or a linear function of $s$ of the form $f(s)= \pm s+b$.

Proof. Suppose that $\gamma$ is of restricted type, then by the use of (19) the equality

$$
\left[\begin{array}{l}
\gamma_{1}^{\prime \prime \prime}(s) \\
\gamma_{2}^{\prime \prime \prime}(s) \\
\gamma_{3}^{\prime \prime \prime}(s)
\end{array}\right]=\left[\begin{array}{ccc}
-c_{11} & 0 & 0 \\
0 & -c_{22} & 0 \\
0 & 0 & -c_{33}
\end{array}\right] \cdot\left[\begin{array}{l}
\gamma_{1}^{\prime}(s) \\
\gamma_{2}^{\prime}(s) \\
\gamma_{3}^{\prime}(s)
\end{array}\right]
$$

holds. Here $\gamma_{i}^{\prime}, \gamma_{i}^{\prime \prime \prime}(s)$ are the first and the third derivatives of $i^{\text {th }}$ component of $\gamma$ and $c_{i i}$ is the entry of the matrix $A$.

From higher order derivatives of $\gamma$ we get

$$
\begin{aligned}
\gamma^{\prime}(s)= & \left(-r \sin s \sin (f(s))+r \cos s \cos (f(s)) f^{\prime}(s), r \cos s \sin (f(s))\right. \\
& \left.+r \sin s \cos (f(s)) f^{\prime}(s),-r \sin (f(s)) f^{\prime}(s)\right)
\end{aligned}
$$


(24)

$$
\begin{aligned}
\gamma^{\prime \prime \prime}(s)= & \left(r \cos s \cos (f(s))\left(f^{\prime \prime \prime}(s)-\left(f^{\prime}(s)\right)^{3}-3 f^{\prime}(s)\right)\right. \\
& +r \sin s \sin (f(s))\left(1+3\left(f^{\prime}(s)\right)^{2}\right)+r \cos s \sin (f(s))\left(-3 f^{\prime}(s) f^{\prime \prime}(s)\right) \\
& +r \sin s \cos (f(s))\left(-3 f^{\prime \prime}(s)\right), r \cos s \cos (f(s))\left(3 f^{\prime \prime}(s)\right) \\
& +r \sin s \sin (f(s))\left(-3 f^{\prime}(s) f^{\prime \prime}(s)\right)+r \cos s \sin (f(s))\left(-1-3\left(f^{\prime}(s)\right)^{2}\right) \\
& +r \sin s \cos (f(s))\left(f^{\prime \prime \prime}(s)-\left(f^{\prime}(s)\right)^{3}-3 f^{\prime}(s)\right), \\
& \left.r \sin (f(s))\left(-f^{\prime \prime \prime}(s)+\left(f^{\prime}(s)\right)^{3}\right)+r \cos (f(s))\left(-3 f^{\prime}(s) f^{\prime \prime}(s)\right)\right) .
\end{aligned}
$$

Using (22), (23) and (24) we have

$$
\begin{aligned}
f^{\prime \prime \prime}(s)-\left(f^{\prime}(s)\right)^{3}-3 f^{\prime}(s)+c_{11} f^{\prime}(s) & =0, \\
1+3\left(f^{\prime}(s)\right)^{2}-c_{11} & =0, \\
-3 f^{\prime}(s) f^{\prime \prime}(s) & =0, \\
-3 f^{\prime \prime}(s) & =0, \\
-1-3\left(f^{\prime}(s)\right)^{2}+c_{22} & =0, \\
f^{\prime \prime \prime}(s)-\left(f^{\prime}(s)\right)^{3}-3 f^{\prime}(s)+c_{22} f^{\prime}(s) & =0, \\
-f^{\prime \prime \prime}(s)+\left(f^{\prime}(s)\right)^{3}-c_{33} f^{\prime}(s) & =0 .
\end{aligned}
$$

From (27) and (28) it can be seen that either $f(s)$ is constant or a linear function of $s$ of the form $f(s)=a s+b$ where $a, b \in \mathbb{R}$. If $f(s)$ is constant, then $f(s)$ is a circle which is of restricted type. If $f(s)$ is a linear function of $s$ of the form $f(s)=a s+b$, then using (25) and (26) we get $c_{11}=1+3 a^{2}=a^{2}+3$. Then $a= \pm 1$ and $c_{11}=4$. Similarly, from (29), (30) and (31) we get $c_{22}=4$ and $c_{33}=1$. So we obtain

$$
A=\left[\begin{array}{lll}
4 & 0 & 0 \\
0 & 4 & 0 \\
0 & 0 & 1
\end{array}\right]
$$

Conversely, if $f(s)=$ const. or $f(s)= \pm s+b$ then it is easy to show that the curve given with the parametrization (21) is of restricted type.

We also get the following result.

Proposition 4. Let $\gamma$ be closed $W$-curve of rank $k$ and of length $2 \pi r$ in $\mathbb{E}^{2 k}$ given by the parametrization (13). Then $\gamma$ is of restricted type. 
Proof. From higher order derivatives of $\gamma$ we get

$$
\begin{aligned}
& \gamma^{\prime}(s)= \frac{1}{\sqrt{k}}\left(-\sin \left(\frac{t_{1} s}{r}\right), \cos \left(\frac{t_{1} s}{r}\right), \ldots,\right. \\
&\left.-\sin \left(\frac{t_{k} s}{r}\right), \cos \left(\frac{t_{k} s}{r}\right)\right) \\
& \gamma^{\prime \prime}(s)=\frac{-1}{\sqrt{k}}\left(\frac{t_{1}}{r} \cos \left(\frac{t_{1} s}{r}\right), \frac{t_{1}}{r} \sin \left(\frac{t_{1} s}{r}\right), \ldots,\right. \\
&\left.\frac{t_{k}}{r} \cos \left(\frac{t_{k} s}{r}\right), \frac{t_{k}}{r} \sin \left(\frac{t_{k} s}{r}\right)\right) \\
& \gamma^{\prime \prime \prime}(s)=\frac{1}{\sqrt{k}}\left(\frac{t_{1}^{2}}{r^{2}} \sin \left(\frac{t_{1} s}{r}\right),-\frac{t_{1}^{2}}{r^{2}} \cos \left(\frac{t_{1} s}{r}\right), \ldots,\right. \\
&\left.\frac{t_{k}^{2}}{r^{2}} \sin \left(\frac{t_{k} s}{r}\right),-\frac{t_{k}^{2}}{r^{2}} \cos \left(\frac{t_{k} s}{r}\right)\right) .
\end{aligned}
$$

So, we have $\gamma^{\prime \prime \prime}(t)=-A \gamma^{\prime}(t)$ where

$$
A=\left[\begin{array}{ccccc}
\frac{t_{1}^{2}}{r^{2}} & 0 & \cdots & 0 & 0 \\
0 & \frac{t_{1}^{2}}{r^{2}} & \cdots & 0 & 0 \\
\vdots & \vdots & \ddots & \vdots & \vdots \\
0 & 0 & \cdots & \frac{t_{k}^{2}}{r^{2}} & 0 \\
0 & 0 & \cdots & 0 & \frac{t_{k}^{2}}{r^{2}}
\end{array}\right] .
$$

Thus $W$-curve is of restricted type.

Example 6. A closed $\mathrm{W}$-curve of rank 4 and of length $2 \pi$ given by the parametrization

$$
\gamma(s)=(\cos m s, \sin m s, \cos n s, \sin n s)
$$

is of restricted type, where $m, n$ are positive integers. From higher order derivatives of $\gamma$ we get $\gamma^{\prime \prime \prime}(t)=-A \gamma^{\prime}(t)$ where

$$
A=\left[\begin{array}{cccc}
m^{2} & 0 & 0 & 0 \\
0 & m^{2} & 0 & 0 \\
0 & 0 & n^{2} & 0 \\
0 & 0 & 0 & n^{2}
\end{array}\right]
$$

Thus $\gamma$ is of restricted type.

Theorem 1 ([7]). Up to rigid motions of $\mathbb{E}^{2}$, a curve in $\mathbb{E}^{2}$ is of restricted type if and only if it is an open portion of one of the following plane curves:

(1) a circle, 
(2) a line,

(3) a curve with equation : $\cos (c x)=e^{-c y}$, where $c \neq 0$,

(4) a curve with equation : $a \sin ^{2}(\sqrt{c} x)+b \sinh ^{2}(\sqrt{c} x)=c$, where $a>$ $b>0, c=a-b$,

(5) a curve with equation : $a \sin ^{2}(\sqrt{c} x)-b \cosh ^{2}(\sqrt{c} x)=c$, where $a>$ $0>b, c=a-b$.

Proposition $5([7])$. Let $\gamma$ be a planar curve. $\gamma$ is of restricted type if and only if the curvature $\kappa$ of $\gamma$ satisfies the following differential equation

$$
\kappa \kappa^{\prime \prime \prime}-\kappa^{\prime} \kappa^{\prime \prime}+4 \kappa^{3} \kappa^{\prime}=0
$$

where the derivatives are taken with respect to the arc length parameter.

\section{REFERENCES}

[1] B.Y. CHEN, Geometry of Submanifolds and Its Applications, Science University of Tokyo, Tokyo, 1981.

[2] B.Y. CHEN, Total mean curvature and submanifolds of finite type, World Scientific, Singapore, 1984.

[3] B.Y. CHEN, A report on submanifolds of finite type, Soochow J. Math., 22 (1996), $117-337$.

[4] B.Y. CHEN, J. DEPREZ, F. DİLLEN, L. VERSTRAELEN AND L. VRANCKEN, Curves of finite type, Geometry and Topology of Submanifolds, II, World Scientific, Singapore, 1990, 76-110.

[5] B.Y. CHEN, M. PETROVIC, On spectral decomposition of immersions of finite type, Bull. Austr. Math. Soc. 44 (1991), 117-129.

[6] B.Y. CHEN, J. DEPREZ, P. VERHEYEN, Immersions with geodesics of 2-type, In: Geometry and Topology of Submanifolds IV, Belgium, 1992.

[7] B.Y. CHEN, F. DİLLEN, L. VERSTRAELEN AND L. VRANCKEN, Submanifolds of restricted type, Journal of Geometry, 46 (1993), 20-32.

[8] B.Y. CHEN, A report on submanifolds of finite type, Soochow J. Math., 22 (1996), $117-337$.

[9] F. KLEIN, S. LIE, Uber diejenigen ebenenen kurven welche durch ein geschlossenes system von einfach unendlich vielen vartauschbaren linearen Transformationen in sich übergehen, Math. Ann., 4 (1871), 50-84.

[10] F. DILLEN, J. PAS, L. VERSTRAELEN, On surfaces of finite type in Euclidean 3-space, Kodai Math. J., 13 (1990), 10-21.

[11] G. ZAFINDRATAFA, Hypersurfaces whose mean curvature function is of finite type, J. Geom., 55 (1996), 182-191.

[12] H. GLUCK, Higher curvatures of curves in Euclidean space, Am. Math. Month., 73 (1966), 699-704. 
[13] O.J. GARAY, An extension of Takahashi's theorem, Geom. Dedicate, 34 (1990), $105-112$.

[14] T. HASANIS, T. VLACHOS, Hypersurfaces of $\mathbb{E}^{n+1}$ satisfying $\Delta x=A x+B$, Jour. Austr. Math. Soc. 53 (1992), 377-384.

\section{BENGÜ (KILIÇ) BAYRAM}

Department of Mathematics

BALIKESIR UNIVERSITY

BALIKESIR

TURKEY

E-mail address: benguk@balikesir.edu.tr

\section{Nergiz ÖNEN}

Department of Mathematics

Çukurova University

ADANA

TURKEY

E-mail address: nonen@cu.edu.tr 